\title{
A LIFTING CHARACTERIZATION OF RFD C*-ALGEBRAS
}

\author{
DON HADWIN*
}

\begin{abstract}
We prove a conjecture of Terry Loring that characterizes separable RFD C*-algebras in terms of a lifting property. In addition we introduce and study generalizations of RFD algebras. If $k$ is an infinite cardinal, we say a $\mathrm{C}^{*}$-algebra is residually less than $k$ dimensional, if the family of representations on Hilbert spaces of dimension less than $k$ separates the points of the algebra. We give characterizations of this property and prove that this class is closed under free products in the nonunital category. For free products in the unital category, the results depend on the cardinal $k$.
\end{abstract}

\section{Introduction}

A $C^{*}$-algebra $\mathscr{A}$ is residually finite dimensional $(R F D)$ if the collection of all finite-dimensional representations of $\mathscr{A}$ separates the points of $\mathscr{A}$; equivalently, if there is a direct sum of finite-dimensional representations of $\mathscr{A}$ with zero kernel. It is clear that every commutative $\mathrm{C}^{*}$-algebra is RFD. ManDuen Choi [4] showed that free group $\mathrm{C}^{*}$-algebras are RFD. Ruy Exel and Terry Loring [6] proved that the free product of two RFD algebras is RFD. Terry Loring [12] proved that projective $\mathrm{C}^{*}$-algebras are RFD. The class of RFD C*-algebras plays an important role in the theory of $\mathrm{C}^{*}$-algebras, e.g., [1], [2], [3], [4], [5], [6], [7], [12], [10].

Suppose $\left\{e_{1}, e_{2}, \ldots\right\}$ is an orthonormal basis for a Hilbert space $H$, and, for each integer $n \geq 1$, let $P_{n}$ be the projection onto $\operatorname{sp}\left(\left\{e_{1}, \ldots, e_{n}\right\}\right)$, let $\mathscr{M}_{n}=P_{n} B\left(\ell^{2}\right) P_{n}$ for $n \geq 1$, and (see Lemma 1) let

$$
\mathscr{B}=\left\{\left\{T_{n}\right\} \in \prod_{n=1}^{\infty} \mathscr{M}_{n}: \exists T \in B\left(\ell^{2}\right) \text { with } T_{n} \rightarrow T(*-\mathrm{SOT})\right\},
$$

and let

$$
\mathscr{J}=\left\{\left\{T_{n}\right\} \in \mathscr{B}: T_{n} \rightarrow 0(*-\mathrm{SOT})\right\} .
$$

\footnotetext{
* The author wishes to thank Tatiana Shulman for bringing Terry Loring's lifting question to his attention, and he wishes to thank the referee for many valuable comments.

Received 30 May 2012.
} 
Then, (see Lemma 1), $\mathscr{B}$ is a unital $\mathrm{C}^{*}$-algebra, $\mathscr{J}$ is a closed ideal in $\mathscr{B}$ and

$$
\pi\left(\left\{T_{n}\right\}\right)=(*-\mathrm{SOT})-\lim _{n \rightarrow \infty} T_{n}
$$

defines a unital surjective $*$-homomorphism from $\mathscr{B}$ to $B(H)$ whose kernel is $\mathscr{J}$. If $\mathscr{A}$ is a separable $\mathrm{C}^{*}$-algebra and every (or even one faithful) representation from $\mathscr{A}$ to $B(H)$ lifts to a representation from $\mathscr{A}$ to $\mathscr{B}$, then it is clear that $\mathscr{A}$ must be RFD.

The idea of using this technique to prove an algebra is RFD first appeared in [7] (see also [12]). It was conjectured by Terry Loring (private communication) that every separable RFD C*-algebra has this lifting property. In this paper we prove Loring's conjecture (Theorem 11).

We also introduce a related notion. Suppose $k$ is an infinite cardinal. We say that a $\mathrm{C}^{*}$-algebra $\mathscr{A}$ is residually less than $k$-dimensional, conveniently denoted by $R_{<k} D$, if the class of representations of $\mathscr{A}$ on Hilbert spaces of dimension less than $k$ separates the points of $\mathscr{A}$; equivalently, if there is a direct sum of such representations that has zero kernel. Note that when $k=\aleph_{0}$, we have $R_{<k} D$ is the same as RFD. We give characterizations of $R_{<k} D$ algebras that show that the free product of an arbitrary collection of $R_{<k} D C^{*}$-algebras is $R_{<k} D$. We also give conditions that ensure that the free product (amalgamated over C) of unital $\mathrm{C}^{*}$-algebras in the category of unital $\mathrm{C}^{*}$-algebras is $R_{<k} D$; this always happens when each of the algebras has a one-dimensional unital representation.

The proofs of all of our results rely on a simple result (Lemma 1) and results of the author [8], [9] on approximate unitary equivalence and approximate summands of nonseparable representations of nonseparable $\mathrm{C}^{*}$-algebras.

Suppose $k$ and $m$ are infinite cardinals. We say that a $\mathrm{C}^{*}$-algebra $\mathscr{A}$ is $m$ generated if it is generated by a set with cardinality at most $m$. For each cardinal $s$, we let $H_{s}$ be a Hilbert space whose dimension is $s$. If $\pi: \mathscr{A} \rightarrow B(H)$ is a $*$-homomorphism, we say that the dimension of $\pi$ is $\operatorname{dim} \pi=\operatorname{dim} H$. We define $\operatorname{Rep}_{\leq k}(\mathscr{A})$ to be the set of all representations $\pi: \mathscr{A} \rightarrow B\left(H_{s}\right)$ for some $s<k$.

If $\mathscr{A}$ is a $\mathrm{C}^{*}$-algebra, then $\mathscr{A}^{+}$denotes the $\mathrm{C}^{*}$-algebra obtained by adding a unit to $\mathscr{A}$ (which is different from the unit in $\mathscr{A}$ if $\mathscr{A}$ is unital).

We end this section with our key lemma. Suppose $H$ is a Hilbert space and $P$ is a projection in $B(H)$. We define $\mathscr{M}_{P}=P B(H) P$. Then $\mathscr{M}_{P}$ is a unital $\mathrm{C}^{*}$ algebra, but the unit is $P$, not 1 . However, $\mathscr{M}_{P}$ is a $C^{*}$-subalgebra of $B(H)$. A unitary element of $\mathscr{M}_{P}$ is an operator $U \in B(H)$ such that $U U^{*}=U^{*} U=P$, and is the direct sum of a unitary operator on $P(H)$ with 0 on $P(H)^{\perp}$. If $P \neq 1$, a unitary operator in $\mathscr{M}_{P}$ is never unitary in $B(H)$.

We use the symbol $*$-SOT to denote the $*$-strong operator topology. 
Lemma 1. Suppose $\left\{P_{\alpha}\right\}$ is a net of projections in $B(H)$ such that $P_{\alpha} \rightarrow 1$ (*-SOT) and let

$$
\mathscr{B}=\left\{\left\{T_{\alpha}\right\} \in \prod_{\alpha} \mathscr{M}_{P_{\alpha}}: \exists T \in B(H), T_{\alpha} \rightarrow T(*-\mathrm{SOT})\right\},
$$

and

$$
\mathscr{J}=\left\{\left\{T_{\alpha}\right\} \in \mathscr{B}: T_{\alpha} \rightarrow 0(*-\mathrm{SOT})\right\},
$$

and define $\pi: \mathscr{B} \rightarrow B(H)$ by

$$
\pi\left(\left\{T_{\alpha}\right\}\right)=(*-\mathrm{SOT})-\lim _{\alpha} T_{\alpha} .
$$

Then

(1) $\mathscr{B}$ is a unital $C^{*}$-algebra,

(2) $\mathscr{J}$ is a closed two-sided ideal in $\mathscr{B}$,

(3) If $T \in B(H)$, then $\left\{P_{\alpha} T P_{\alpha}\right\} \in \mathscr{B}$ and $\pi\left(\left\{P_{\alpha} T P_{\alpha}\right\}\right)=T$,

(4) $\pi$ is a unital surjective $*$-homomorphism

(5) If $U \in B(H)$ is unitary, then there is a unitary $\left\{U_{\alpha}\right\} \in \mathscr{B}$ such that

$$
\pi\left(\left\{U_{\alpha}\right\}\right)=U
$$

Proof. Statements (1)-(4) are easily proved. To prove (5), note that if $U \in B(H)$ is unitary, then there is an $A=A^{*} \in B(H)$ such that $U=e^{i A}$. Using (3), we can easily choose $A_{\alpha}=A_{\alpha}^{*}$ for each $\alpha$ so that $\pi\left(\left\{A_{\alpha}\right\}\right)=A$. Thus, if $U_{\alpha}=e^{i A_{\alpha}}$ (in $\mathscr{M}_{P_{\alpha}}$ ), then $\left\{U_{\alpha}\right\}$ is unitary in $\mathscr{B}$ and $\pi\left(\left\{U_{\alpha}\right\}\right)=U$.

Here is a simple application that gives the flavor of our results.

COROLlaRY 2. Every free group is RFD.

Proof. Suppose $\mathrm{F}$ is a free group and $\mathscr{A}=\mathscr{C}^{*}(\mathrm{~F})=C^{*}\left(\left\{U_{g}: g \in \mathrm{F}\right\}\right)$. Choose a Hilbert space $H$ and a faithful representation $\rho: \mathscr{A} \rightarrow B(H)$. Choose a net $\left\{P_{\alpha}\right\}$ of finite-rank projections such that $P_{\alpha} \rightarrow 1$ (*-SOT). Applying Lemma 1 we have, for each $g \in \mathrm{F}$, we can find a unitary element $\left\{U_{g, \alpha}\right\}$ in $\mathscr{B}$ so that $\pi\left(\left\{U_{g, \alpha}\right\}\right)=U_{g}$. For each $\alpha$, we have a unitary group representation $\sigma_{\alpha}: \mathrm{F} \rightarrow \mathscr{M}_{P_{\alpha}}$ defined by

$$
\sigma_{\alpha}(g)=U_{g, \alpha} .
$$

By the definition of $C^{*}(\mathrm{~F})$, there is a $*$-homomorphism $\tau_{\alpha}: \mathscr{A} \rightarrow \mathscr{M}_{\alpha}$ such that $\tau_{\alpha}\left(U_{g}\right)=U_{g, \alpha}$. It follows that $\tau: \mathscr{A} \rightarrow \mathscr{B}$ defined by $\tau\left(U_{g}\right)=\left\{U_{g, a}\right\}$ is 
a $*$-homomorphism such that $\pi \circ \tau=\rho$. Hence the direct sum of the $\tau_{\alpha}$ 's is faithful, which shows that $\mathscr{A}$ is $R F D$.

The following corollary is from [3, Exercise 7.1.4].

COROLlary 3. Every $C^{*}$-algebra is a $*$-homomorphic image of an RFD $C^{*}$-algebra.

Proof. Suppose $\mathscr{A}$ is a $\mathrm{C}^{*}$-algebra. We can assume that $A \subseteq B(H)$ for some Hilbert space $H$. Choose a net $\left\{P_{\alpha}\right\}$ of finite-rank projections converging *-strongly to 1 , and let $\mathscr{B}, \mathscr{J}$ and $\pi$ be as in Lemma 1 . Then $\mathscr{B}$, and thus $\pi^{-1}(\mathscr{A})$, is $R F D$ and $\pi\left(\pi^{-1}(\mathscr{A})\right)=\mathscr{A}$.

\section{2. $R_{<k} D$ Algebras}

We now prove our main results on $R_{<k} D \mathrm{C}^{*}$-algebras. The following two lemmas contain the key tools.

Lemma 4. Suppose $\aleph_{0} \leq k \leq m$, and $\mathscr{A}$ is $R_{<k} D$ and m-generated. Then

(1) We can write $H_{m}=\sum_{\lambda \in \Lambda}^{\oplus} X_{\lambda}$ with Card $\Lambda=m$, and such that, for every $\lambda \in \Lambda, \operatorname{dim} X_{\lambda}<k$ and there is a unital representation $\pi_{\lambda}$ : $\mathscr{A}^{+} \rightarrow B\left(X_{\lambda}\right)$ such that the representation $\pi: \mathscr{A}^{+} \rightarrow B\left(H_{m}\right)$ defined by $\pi=\sum^{\oplus} \pi_{\lambda}$ is faithful. Moreover, this can be done so that, for each $\lambda_{0} \in \Lambda$, we have $\operatorname{Card}\left(\left\{\lambda \in \Lambda: \pi_{\lambda} \approx \pi_{\lambda_{0}}\right\}\right)=m$.

(2) It is possible to choose the decomposition in (1) so that, for each cardinal $s<k$, there is a $\lambda \in \Lambda$ such that $\operatorname{dim} X_{\lambda}=s$.

Proof. Since $\mathscr{A}$ is $R_{<k} D$, there is a direct sum of representations in $\operatorname{Rep}_{\leq k}(\mathscr{A})$ whose direct sum is faithful. Suppose $D$ is a generating set for $\mathscr{A}$ and $\operatorname{Card}(D) \leq m$. We can replace $D$ by the $*$-algebra over $\mathrm{Q}+i \mathrm{Q}$ generated by $D$ making the cardinality exceed $m$. For each $a \in D$ we can choose a direct sum of countably many summands from our faithful direct sum that preserves the norm of $a$. Hence, by choosing $\aleph_{0} \operatorname{Card}(D)$ summands, we get a direct sum that is isometric on $D$ and thus isometric on $\mathscr{A}$. Since $\aleph_{0} \operatorname{Card}(D) \leq m$. we can replace this last direct sum with a direct sum of $m$ copies of itself and get a direct sum on a Hilbert space with dimension $m$. We can replace this Hilbert space with $H_{m}$ and get a decomposition as in (1). To get (2), note that, since $\mathscr{A}^{+}$has a unital one-dimensional representation, we know that, for every cardinal $s<k$, there is a representation of $\mathscr{A}^{+}$of dimension $s$. If we take one such representation for each $s<k$ and take a direct sum of $m$ copies of all of them, we get a representation that has has dimension at most $m$, so we add this as a summand to the representation we constructed satisfying (1).

Lemma 5. Suppose $\mathscr{A}$ is a $C^{*}$-algebra, $k \leq m$ are infinite cardinals and $D$ is a generating set for $\mathscr{A}$. Suppose we can write $H_{m}=\sum_{\lambda \in \Lambda}^{\oplus} X_{\lambda}$ and 
$\pi=\sum^{\oplus} \pi_{\lambda}$ as in part (1) of Lemma 4 so that $\operatorname{Card}\left(\left\{\lambda \in \Lambda: \pi_{\lambda} \approx \pi_{\lambda_{0}}\right\}\right)=m$ for each $\lambda_{0} \in \Lambda$. If $\rho: \mathscr{A}^{+} \rightarrow B\left(H_{m}\right)$ is a unital representation, then, for every $\varepsilon>0$, every finite subset $J \subseteq D$ and every finite subset $E \subseteq H_{m}$, there is a finite subset $F \subseteq \Lambda$, such that, for every finite set $G$ with $F \subseteq G \subseteq \Lambda$, if $Q_{G}$ is the orthogonal projection onto $\sum_{\lambda \in G}^{\oplus} X_{\lambda}$, then there is a unitary $U \in Q_{G} B\left(H_{m}\right) Q_{G}$ such that, for every $a \in J$ and $e \in E$, we have

$$
\left\|\left[\rho(a)-U_{G}^{*} \pi(a) U_{G}\right] e\right\|=\left\|\left[\rho(a)-U_{G}^{*}\left(\sum_{\lambda \in G} \pi_{\lambda}\right)(a) U_{G}\right] e\right\|<\varepsilon .
$$

Proof. Let $\operatorname{Rep}\left(\mathscr{A}, H_{m}\right)$ denote the set of all unital representations from $\mathscr{A}$ into $B\left(H_{m}\right)$ topologized by the topology of pointwise $*$-SOT convergence, and let $\mathscr{S}$ denote the closure of the elements in $\operatorname{Rep}\left(\mathscr{A}, H_{m}\right)$ that are unitarily equivalent to $\pi$. We want to prove that $\rho \in \mathscr{S}$. It follows that if $a \in \mathscr{A}$ and $a \neq 0$, then $\operatorname{rank} \pi(a)=m=\operatorname{rank}(\pi \oplus \rho)(a)$. Hence, by [8], $\pi$ is approximately unitarily equivalent to $\pi \oplus \rho$. Hence there is a unitary $X$ such that $X^{*}(\pi \oplus \rho) X \in \mathscr{S}$. However, by [8] (and ideas in [9]), $\rho$ is a point*-SOT limit of representations unitarily equivalent to $\pi \oplus \rho$. Indeed, if for each finite subset $\alpha$ of $H_{m}, U_{\alpha}: H_{m} \rightarrow H_{m} \oplus H_{m}$ is a unitary operator such that $U_{\alpha} x=0 \oplus x$ for each $x \in \alpha$, we have $\left\{U_{\alpha}\right\}$ forms a net such that $\left\{U_{\alpha}^{*}(\pi \oplus \rho)(a) U_{\alpha}\right\}$ converges $*$-strongly to $\rho(a)$ for every $a \in \mathscr{A}$. Thus $W_{\alpha}=X^{*} U_{\alpha}$ is a unitary in $B\left(H_{m}\right)$ for each $\alpha$, and, for every $a \in \mathscr{A}$,

$$
(*-\mathrm{SOT}) \lim _{\alpha} W_{\alpha}^{*}\left[X^{*}(\pi \oplus \rho)(a) X\right] W_{\alpha}=\rho(a) .
$$

Hence, $\rho \in \mathscr{S}$.

Thus there is a net $\left\{V_{\lambda}\right\}$ of unitaries in $B\left(H_{m}\right)$ such that $\left\{V_{\lambda}^{*} \pi(\cdot) V_{\lambda}\right\}$ converges pointwise $*$-strongly to $\rho$. However, the net $\left\{Q_{F}: F \subseteq \Lambda, F\right.$ is finite $\}$ is a net of projections converging $*$-strongly to 1 . Hence, by Lemma 1 , each $V_{\lambda}$ is a $*$-SOT limit of unitaries in the union of $Q_{F} B\left(H_{m}\right) Q_{F}(F \subseteq \Lambda, F$ is finite). The result now easily follows.

THEOREM 6. Suppose $\aleph_{0} \leq k \leq m$, and $\mathscr{A}$ is $m$-generated with a generating set $\mathscr{G}$ with $\operatorname{Card} \mathscr{G} \leq m$. The following are equivalent.

(1) $\mathscr{A}$ is $R_{<k} D$.

(2) There is a faithful unital *-homomorphism $\rho: \mathscr{A}^{+} \rightarrow B\left(H_{m}\right)$ such that, for every $\varepsilon>0$, every finite subset $E \subseteq H_{m}$ and every finite subset $W \subseteq \mathscr{G}$, there is a projection $P \in B\left(H_{m}\right)$ and a unital $*$-homomorphism $\tau: \mathscr{A} \rightarrow M_{P}=P B\left(H_{m}\right) P$ such that, for everye $\in E$ and every $a \in W$ we have

$$
\|[\tau(a)-\rho(a)] e\|<\varepsilon .
$$


(3) There is a faithful unital representation $\rho: \mathscr{A}^{+} \rightarrow B\left(H_{m}\right)$ and a net $\left\{P_{\alpha}\right\}$ of projections in $B\left(H_{m}\right)$, each with rank less than $k$, such that $P_{\alpha} \rightarrow 1$ (*-SOT) and such that, for each $\alpha$, there is a representation $\pi_{\alpha}: \mathscr{A} \rightarrow \mathcal{M}_{P_{\alpha}}$ such that, for every a $\in \mathscr{A}$, we have

$$
\pi_{\alpha}(a) \rightarrow \rho(a)(*-\mathrm{SOT})
$$

(4) For every unital representation $\rho: \mathscr{A}^{+} \rightarrow B\left(H_{m}\right)$ there is a net $\left\{P_{\alpha}\right\}$ of projections in $B\left(H_{m}\right)$, each with rank less than $k$, such that $P_{\alpha} \rightarrow 1$ (*-SOT) and such that, for each $\alpha$, there is a representation $\pi_{\alpha}: \mathscr{A} \rightarrow$ $\mathcal{M}_{P_{\alpha}}$ such that, for every $a \in \mathscr{A}$, we have

$$
\pi_{\alpha}(a) \rightarrow \rho(a)(*-\mathrm{SOT})
$$

Proof. (2) $\Rightarrow(1)$ Let $A$ be the set of triples $(\varepsilon, E, W)$ ordered by $(\geq, \subseteq, \subseteq)$. If $\alpha=(\varepsilon, E, W)$ let $\tau_{\alpha}: \mathscr{A} \rightarrow P_{\alpha} B\left(H_{m}\right) P_{\alpha}$ guaranteed by (2). Since $\mathscr{G}=\mathscr{G}^{*}$ we have

$$
(*-\mathrm{SOT}) \lim _{\alpha} \tau_{\alpha}(a)=\rho(a)
$$

for every $a \in \mathscr{G}$. Since $\rho$ and each $\tau_{\alpha}$ is a $*$-homomorphism, the set of $a \in \mathscr{A}$ for which $\left(*\right.$-SOT) $\lim _{\alpha} \tau_{\alpha}(a)=\rho(a)$ is a unital $\mathrm{C}^{*}$-algebra and is thus $\mathscr{A}^{+}$. Hence, for every $a \in \mathscr{A}^{+}$, we have

$$
\|a\|=\|\rho(a)\| \leq \sup \left\{\left\|\tau_{\alpha}(a)\right\|: \alpha \in A\right\}
$$

Therefore the direct sum of the $\tau_{\alpha}$ 's is faithful and (1) is proved.

$(3) \Rightarrow(2)$. This is obvious.

$(4) \Rightarrow(3)$. It is clear that we need only show that there is a faithful unital representation $\rho: \mathscr{A}^{+} \rightarrow B\left(H_{m}\right)$. Suppose $\tau: \mathscr{A}^{+} \rightarrow B(M)$ is an irreducible representation, and suppose $D$ is a generating set with $\operatorname{Card}(D) \leq m$. Let $\mathscr{A}_{0}$ be the unital $*$-subalgebra of $\mathscr{A}^{+}$over the field $\mathrm{Q}+i \mathrm{Q}$ of complex rational numbers. Then $\mathscr{A}_{0}$ is norm dense in $\mathscr{A}$ and Card $\mathscr{A}_{0}=$ Card $D \leq m$. Suppose $f \in M$ is a unit vector. Since $\tau$ is irreducible, $\tau\left(\mathscr{A}_{0}\right) f$ must be dense in $M$. Suppose $B$ is an orthonormal basis for $M$, and, for each $e \in B$ let $U_{e}$ be the open ball centered at $e$ with radius $\sqrt{2} / 2$. Each $U_{e}$ must intersect the dense set $\tau\left(\mathscr{A}_{0}\right) f$, and since the collection $\left\{U_{e}: e \in B\right\}$ is disjoint, we conclude that

$$
\operatorname{dim} M=\operatorname{Card} B \leq \operatorname{Card} \tau\left(\mathscr{A}_{0}\right) f \leq \operatorname{Card}\left(\mathscr{A}_{0}\right) \leq m
$$

We know that for every $x \in \mathscr{A}_{0}$ there is an irreducible representation $\tau_{x}$ : $\mathscr{A}^{+} \rightarrow B\left(M_{x}\right)$ such that $\left\|\tau_{x}(x)\right\|=\|x\|$. Since $\operatorname{dim} \sum_{x \in \mathscr{A}_{0}}^{\oplus} M_{x} \leq m \cdot m=m$, there is a representation $\rho: \mathscr{A}^{+} \rightarrow B\left(H_{m}\right)$ that is unitarily equivalent to a 
direct sum of $m$ copies of $\sum_{x \in \mathscr{A}_{0}}^{\oplus} \tau_{x}$. Hence $\rho$ is isometric on the dense subset $\mathscr{A}_{0}$, which implies $\rho$ is faithful.

(1) $\Rightarrow$ (3). Since $\mathscr{A}$ is $R_{<k} D$, we can choose a decomposition $H_{m}=$ $\sum_{\lambda \in \Lambda}^{\oplus} X_{\lambda}$ and representation $\pi=\sum_{\lambda \in \Lambda}^{\oplus} \pi_{\lambda}$ as in part (1) of Lemma 4. Now (3) follows from Lemma 5.

We see that the class of $R_{<k} D$ algebras is closed under arbitrary free products in the nonunital category of $\mathrm{C}^{*}$-algebras.

THeOREm 7. Suppose $k$ is an infinite cardinal and $\left\{\mathscr{A}_{l}: i \in I\right\}$ is a family of $R_{<k} D C^{*}$-algebras. Then the free product $* \mathscr{A}_{i}$ is $R_{<k} D$.

Proof. Choose an infinite cardinal $m \geq k+\sum_{i \in I} \operatorname{Card}\left(\mathscr{A}_{i}\right)$. Since $*_{i \in I} \mathscr{A}_{i}$ is generated by $\mathscr{G}=\left[\bigcup_{i \in I} \mathscr{A}_{i}\right] \backslash\{0\} \subseteq *_{i \in I} \mathscr{A}_{i}$, clearly $*_{i \in I} \mathscr{A}_{i}$ is $m$-generated. Choose a set $\Lambda$ with $\operatorname{Card}(\Lambda)=m$ and let $S$ be the set of cardinals less than $k$. Write

$$
H_{m}=\sum_{s \in S}^{\oplus} \sum_{\lambda \in \Lambda} X_{s, \lambda}
$$

where $\operatorname{dim} X_{s, \lambda}=s$ for every $s \in S$ and $\lambda \in \Lambda$. It follows that, for each $i \in I$, we can find a representation $\pi^{i}: \mathscr{A}_{i} \rightarrow B\left(H_{m}\right)$ such that

$$
\pi^{i}=\sum_{s \in S}^{\oplus} \sum_{\lambda \in \Lambda} \pi_{s, \lambda}^{i}
$$

satisfying (1) and (2) of Lemma 4. Suppose $\varepsilon>0, E \subseteq H_{m}$ is finite and $W \subseteq \mathscr{G}$ is finite. We can write $W$ as a disjoint union of $W_{i_{1}}, \ldots, W_{i_{n}}$ with $W_{i}=W \cap \mathscr{A}_{i}$. Let $\rho_{i}$ be the restriction of $\rho$ to $\mathscr{A}_{i}$. Applying Lemma 5 to $\mathscr{A}_{i_{j}}$ and $\rho_{i_{j}}$ and $\pi^{i_{j}}$ for $1 \leq j \leq n$, we can find one finite subset $G \subseteq S \times \Lambda$ so that if $P$ is the projection on $\sum_{(s, \lambda) \in G}^{\oplus} X_{s, \lambda}$, then there are unitary operators $U_{i_{1}}, \ldots, U_{i_{n}} \in M_{P}=P B\left(H_{m}\right) P$ so that, for $1 \leq j \leq n, a \in W_{j}, e \in E$, we have

$$
\left\|\left[\rho_{i_{j}}(a)-U_{i j}^{*} \pi^{i_{j}}(a) U_{i j}\right] e\right\|<\varepsilon .
$$

Define $\tau_{i j}: \mathscr{A}_{i_{j}}^{+} \rightarrow \mathscr{M}_{P}$ by

$$
\tau_{i j}(a)=U_{i j}^{*} \pi^{i_{j}}(a) U_{i j},
$$

and for $i \in I \backslash\left\{i_{1}, \ldots, i_{n}\right\}$ define $\tau_{i}: \mathscr{A}_{i} \rightarrow \mathscr{M}_{P}$ by

$$
\tau_{i}(a)=P \pi^{i}(a) P .
$$

Then, by the definition of free product, there is a representation $\tau: *_{i \in I} \mathscr{A}_{i}^{+} \rightarrow$ $\mathscr{M}_{P}$ such that $\tau \mid \mathscr{A}_{i}=\tau_{i}$ for every $i \in I$. It follows that, for every $e \in E$ and 
every $a \in W$,

$$
\|[\rho(a)-\tau(a)] e\|<\varepsilon .
$$

It follows from part (2) of Theorem 6 that $*_{i \in I} \mathscr{A}_{i}$ is $R_{<k} D$.

Corollary 8. Suppose $k$ is an infinite cardinal and $\left\{\mathscr{A}_{l}: i \in I\right\}$ is a family of $R_{<k} D C^{*}$-algebras such that each $\mathscr{A}_{i}$ has a one-dimensional unital representation. Then the unital free product $*_{c_{i \in I}} \mathscr{A}_{i}$ is $R_{<k} D$.

Proof. This follows from the fact that if $\tau_{i}: \mathscr{A}_{i} \rightarrow \mathrm{C}$ is a unital $*-$ homomorphism for each $i \in I$, then $*_{c_{i \in I}} \mathscr{A}_{i}$ is $*$-isomorphic to $\left(*_{i \in I} \operatorname{ker} \tau_{i}\right)^{+}$.

Without the condition on unital one-dimensional representations, the preceding corollary may fail. For example, $*_{c_{n \in \mathrm{N}}} \mathcal{M}_{n}(\mathrm{C})$ is not $R F D\left(=R_{<\aleph_{0}} D\right)$, even though each $\mathscr{M}_{n}(\mathrm{C})$ is $R F D$. The reason is that each unital representation of the free product must be injective on each $\mathscr{M}_{n}(\mathrm{C})$ and must have infinitedimensional range.

However, there is something we can say about the general situation. If $k$ is a limit cardinal (i.e., $k$ is the supremum of all the cardinals less than $k$ ), the cofinality of $k$ is the smallest cardinal $s$ for which there is a set $E$ of cardinals less than $k$ whose supremum is $k$. Clearly, the cofinality of $k$ is at most $k$. If $k$ is not a limit cardinal, then there is a cardinal $s$ such that $k$ is the smallest cardinal larger than $s$, and if $E$ is a set of cardinals less than $k$, then $\sup (E) \leq s<k$.

Theorem 9. Suppose $k$ is an infinite cardinal and $\left\{\mathscr{A}_{l}: i \in I\right\}$ is a family of unital $R_{<k} D C^{*}$-algebras. Then

(1) If $k$ is a limit cardinal and $\operatorname{Card}(I)$ is less than the cofinality of $k$, then the free product $*_{c i \in I} \mathscr{A}_{i}$ is $R_{<k} D$.

(2) If $k$ is not a limit cardinal, then the free product $*_{c_{i \in I}} \mathscr{A}_{i}$ is $R_{<k} D$.

Proof. (1). Choose $m \geq k+\sum_{i \in I} \operatorname{Card}\left(\mathscr{A}_{i}\right)$, and choose a set $\Lambda$ with $\operatorname{Card}(\Lambda)=m$. Using Lemma 4 we can, for each $i \in I$, find a faithful representation $\pi^{i}=\sum_{\lambda \in \Lambda} \pi_{\lambda, i}$ so that $\operatorname{dim} \pi^{i}=m$ and, for every $i \in I$ and $\lambda \in \Lambda$, we have $\operatorname{dim} \pi_{\lambda, i}<k$. Since $\operatorname{Card}(I)$ is less than the cofinality of $k$, we have, for each $\lambda \in \Lambda$, a cardinal $s_{\lambda}<k$ such that $\sup _{i \in I} \operatorname{dim} \pi_{\lambda, i} \leq s_{\lambda}$. If we replace each $\pi_{\lambda, i}$ with a direct sum of $s_{\lambda}$ copies of itself, we get a new decomposition which we will denote by the same names such that, for each $i$ and each $\lambda$ we have $\operatorname{dim} \pi_{\lambda, i}=s_{\lambda}$. Hence we may write direct sum decompositions of the $\pi^{i}$,s with respect to a common decomposition $H_{m}=\sum_{\lambda \in \Lambda} X_{\lambda}$ where $\operatorname{dim} X_{\lambda}=s_{\lambda}$ for every $\lambda \in \Lambda$. The rest now follows as in the proof of Lemma 7.

(2). If $k$ is not a limit cardinal, there is a largest cardinal $s<k$. Repeat the proof of part (1) with $s_{\lambda}=s$ for every $\lambda \in \Lambda$.

REMARK 10. We cannot remove the condition on Card $(I)$ in part (1) of Theorem 9. Suppose $k$ is a limit cardinal and $I$ is a set of cardinals less than $k$ 
whose cardinality equals the cofinality of $k$ and such that $\sup (I)=k$. For each infinite cardinal $m$, choose a set $\Lambda_{m}$ with cardinality $m$, and let $\mathscr{S}_{m}$ denote the universal unital $\mathrm{C}^{*}$-algebra generated by $\left\{v_{\lambda}: \lambda \in \Lambda_{m}\right\}$ with the conditions

(1) $v_{\lambda}^{*} v_{\lambda}=1$ for every $\lambda \in \Lambda_{m}$,

(2) $v_{\lambda_{1}} v_{\lambda_{1}}^{*} v_{\lambda_{2}} v_{\lambda_{2}}^{*}=0$ for $\lambda_{1} \neq \lambda_{2}$ in $\Lambda_{m}$.

Since $\mathscr{S}_{m}$ is $m$-generated, it follows that every irreducible representation of $\mathscr{S}_{m}$ is at most $m$-dimensional (see the proof of (4) $\Rightarrow$ (3) in Theorem 6). Hence $\mathscr{S}_{m}$ is separated by $m$-dimensional representations. On the other hand, if $\pi$ is a unital representation of $\mathscr{S}_{m}$, then $\left\{\pi\left(v_{\lambda ;} v_{\lambda}^{*}\right): \lambda \in \Lambda_{m}\right\}$ is an orthogonal family of nonzero projections, which implies that the dimension of $\pi$ is at least $m$.It follows that each $\mathscr{S}_{s}$ is $R_{<k} D$ for $s \in I$. However, any unital representation $\pi$ of the free product $*_{c_{s} \in I} \mathscr{S}_{s}$ must induce a unital representation of each $\mathscr{S}_{s}$, so its dimension is at least $\sup I=k$. Hence $*_{c_{s} \in I} \mathscr{S}_{s}$ is not $R_{<k} D$.

\section{Separable RFD Algebras}

In this section we show that for a separable $\mathrm{C}^{*}$-algebra being RFD is equivalent to a lifting property.

Suppose $\left\{e_{1}, e_{2}, \ldots\right\}$ is an orthonormal basis for a Hilbert space $\ell^{2}$, and, for each integer $n \geq 1$, let $P_{n}$ be the projection onto $\operatorname{sp}\left(\left\{e_{1}, \ldots, e_{n}\right\}\right)$. Let $\mathscr{M}_{n}=P_{n} B\left(\ell^{2}\right) P_{n}$ for $n \geq 1$, and, following Lemma 1, let

$$
\mathscr{B}=\left\{\left\{T_{n}\right\} \in \prod_{n=1}^{\infty} \mathscr{M}_{n}: \exists T \in B\left(\ell^{2}\right) \text { with } T_{n} \rightarrow T(*-\mathrm{SOT})\right\},
$$

and let

$$
\mathscr{J}=\left\{\left\{T_{n}\right\} \in \mathscr{B}: T_{n} \rightarrow 0(*-\mathrm{SOT})\right\} .
$$

Then, by Lemma 1 , we have that $\mathscr{B}$ is a unital $\mathrm{C}^{*}$-algebra, $\mathscr{J}$ is a closed ideal in $\mathscr{B}$ and

$$
\pi\left(\left\{T_{n}\right\}\right)=(*-\mathrm{SOT})-\lim _{n \rightarrow \infty} T_{n}
$$

defines a unital surjective $*$-homomorphism from $\mathscr{B}$ to $B(H)$ whose kernel is $\mathscr{J}$. We can now give our characterization of RFD for separable $\mathrm{C}^{*}$-algebras.

Theorem 11. Suppose $\mathscr{A}$ is a separable $C^{*}$-algebra. The following are equivalent.

(1) $\mathscr{A}$ is RFD

(2) For every unital $*$-homomorphism $\rho: \mathscr{A}^{+} \rightarrow B\left(\ell^{2}\right)$ there is a unital $*$-homomorphism $\tau: \mathscr{A}^{+} \rightarrow \mathscr{B}$ such that $\pi \circ \tau=\rho$.

PRoof. The implication (2) $\Rightarrow(1)$ is clear. 
$(1) \Rightarrow(2)$. Suppose $\mathscr{A}=\mathscr{C}^{*}\left(\left\{a_{1}, a_{2}, \ldots\right\}\right)$ is $R F D$ and $\rho: \mathscr{A}^{+} \rightarrow B\left(\ell^{2}\right)$ is a unital $*$-homomorphism. It follows from Theorem 6 that there is an increasing sequence $\left\{n_{k}\right\}$ of positive integers and unital $*$-homomorphisms $\tau_{k}: \mathscr{A} \rightarrow$ $\mathcal{M}_{n_{k}}$ such that

$$
\left\|\left[\tau_{k}\left(a_{j}\right)-\rho\left(a_{j}\right)\right] e_{i}\right\|<1 / k
$$

for $1 \leq i, j \leq k$. It follows that $\tau_{n_{k}}(a) \rightarrow \rho(a)(*-S O T)$ for every $a \in \mathscr{A}^{+}$. If $n_{k}<n<n_{k+1}$ we define $\tau_{n}: \mathscr{A}^{+} \rightarrow \mathscr{M}_{n}$ by

$$
\tau_{n}(a)=\left(\begin{array}{llll}
\tau_{n_{k}}(a) & & & \\
& \beta(a) & & \\
& & \ddots & \\
& & & \beta(a)
\end{array}\right)
$$

where $\beta: \mathscr{A}^{+} \rightarrow \mathrm{C}$ is the unique $*$-homomorphism with $\operatorname{ker} \beta=\mathscr{A}$, relative to the decomposition

$$
P_{n}\left(\ell^{2}\right)=P_{n_{k}}\left(\ell^{2}\right) \oplus \mathrm{C} e_{1+n_{k}} \oplus \cdots \oplus \mathrm{C} e_{-1+n_{k+1}} .
$$

It is easily seen that $\tau_{n}(a) \rightarrow \rho(a)\left(*-\right.$ SOT) for every $a \in \mathscr{A}^{+}$. If we define $\tau: \mathscr{A} \rightarrow \mathscr{B}$ by

$$
\tau(a)=\left\{\tau_{n}(a)\right\}
$$

we see that $\pi \circ \tau=\rho$.

\section{REFERENCES}

1. Archbold, R. J.,On residually finite-dimensional $C^{*}$-algebras, Proc. Amer. Math. Soc. 123 (1995), 2935-2937.

2. Brown, N. P.,On quasidiagonal $C^{*}$-algebras, arXiv: math/0008181 (2000).

3. Brown, N. P., and Ozawa, N., $C^{*}$-algebras and finite-dimensional approximations, Grad. Stud. in Math. 88, Amer. Math. Soc., Providence (2008).

4. Choi, M.-D., The full $C^{*}$-algebra of the free group on two generators, Pacific J. Math 87 (1980), 41-48.

5. Dadarlat, M., On the approximation of quasidiagonal $C^{*}$-algebras, J. Funct. Anal. 167 (1999), 69-78.

6. Exel, R., and Loring, T., Finite-dimensional representations of free product $C^{*}$-algebras, Internat. J. Math. 3 (1992), 469-476.

7. Goodearl, K. R., and Menal, P., Free and residually finite-dimensional $C^{*}$-algebras, J. Funct. Anal. 90 (1990), 391-410.

8. Hadwin, D., Nonseparable approximate equivalence, Trans. Amer. Math. Soc. 266 (1981), 203-231.

9. Hadwin, D., An operator-valued spectrum, Indiana Univ. Math. J. 26 (1977), 329-340.

10. Hadwin, D., Li, Q., Li, W., and Shen, J., MF-traces and topological free entropy dimension, preprint.

11. Lin, H., Residually finite-dimensional and AF-embeddable $C^{*}$-algebras, Proc. Amer. Math. Soc. 129 (2001), 1689-1696. 
12. Loring, T., Lifting solutions to perturbing problems in $C^{*}$-algebras, Fields Inst. Mon. 8, Amer. Math. Soc., Providence 1997.

\author{
DEPARTMENT OF MATHEMATICS AND STATISTICS \\ UNIVERSITY OF NEW HAMPSHIRE \\ DURHAM, NH, 03824 \\ USA \\ E-mail: don@math.unh.edu
}

